\title{
Instability and Bifurcation
}

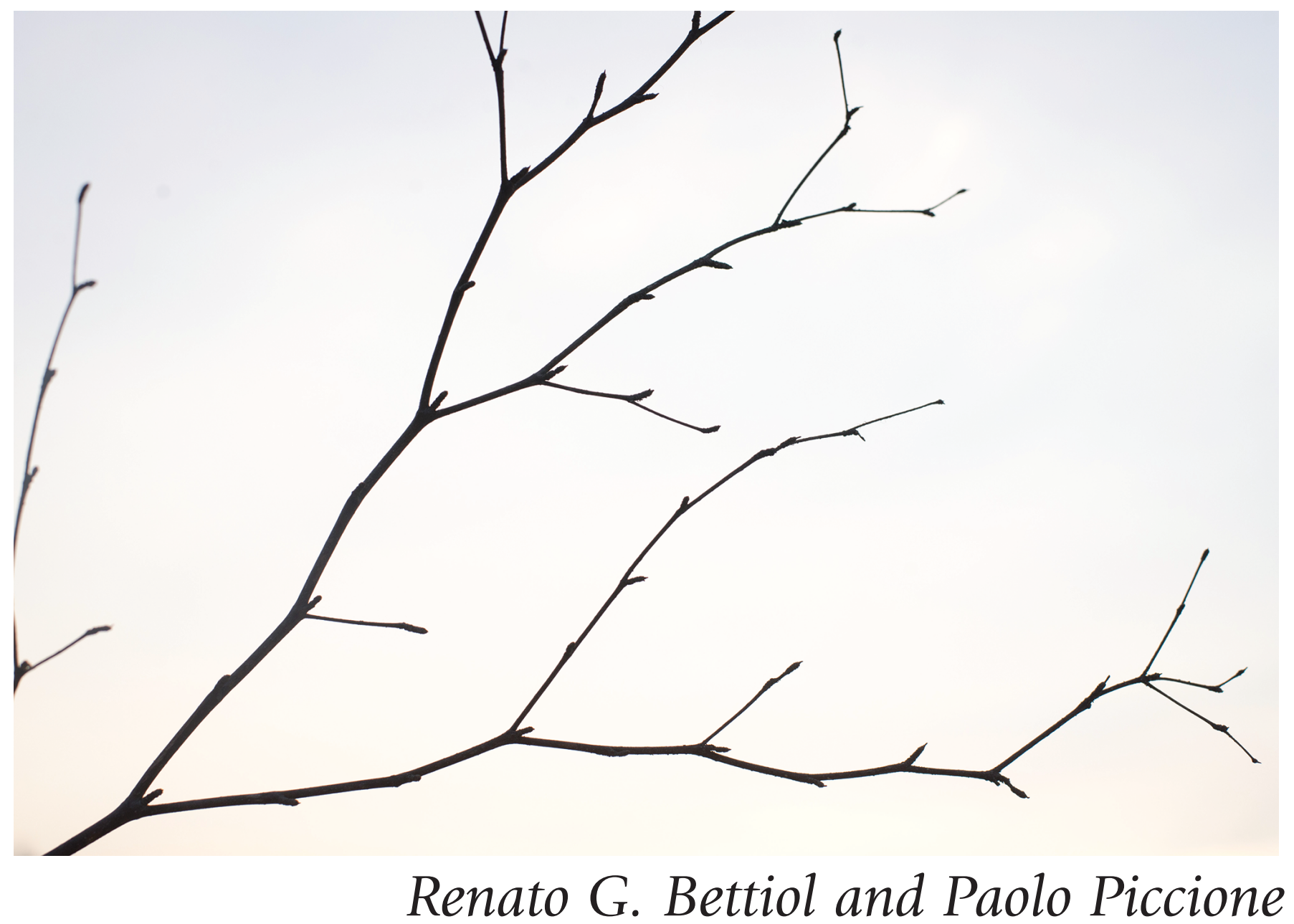

By the Principle of Least Action, physical systems governed by conservative forces typically assume energy-minimizing states. As such, these ground states are stable stationary points of the corresponding energy functional. On the other hand, unstable stationary points may also have very interesting features from a mathematical viewpoint, although (or, perhaps, because) they are harder to find in nature.

Renato G. Bettiol is an assistant professor of mathematics at CUNY, Lehman College. His email address is $\mathrm{r}$. bettiol@1ehman. cuny. edu.

Paolo Piccione is a professor of mathematics at the University of São Paulo, Brazil, president of the Brazilian Mathematical Society, and member of the IMU Executive Committee. His email address is piccione@ime.usp.br.

Communicated by Notices Associate Editor Chikako Mese.

For permission to reprint this article, please contact:

reprint-permission@ams.org.

DOI: https://doi.org/10.1090/noti2185
If the way energy is measured depends on a parameter, a family of stationary points may lose stability when that parameter crosses a certain threshold. Remarkably, this loss of stability creates a new branch of stationary points that splits from the family. This phenomenon was first explored by Poincaré [Poi85], who called it a bifurcation, marking the dawn of a multifaceted theory with applications to Dynamical Systems, Analysis, PDEs, and, more recently, to Differential Geometry and Geometric Analysis.

In this article, we give an overview of classical results in variational Bifurcation Theory and some geometric applications, including multiplicity results for Geodesics, Constant Mean Curvature Surfaces, and the Yamabe problem. These are obtained by exploiting the growing instability of families of trivial (often highly symmetric) solutions as they degenerate. The resulting bifurcating solutions are often less symmetric, and give rise to interesting examples where ground states need not be the most symmetric ones. 


\section{Euler's Buckling Problem}

One of the most classical examples of bifurcation comes from engineering, by subjecting a rod, or column, to compressive stress. The parameter in this case is the amount $P$ of compressive force exerted on the rod (called load), and determining the maximum load $P_{\max }$ that can be supported without structural failures is, evidently, of paramount importance.

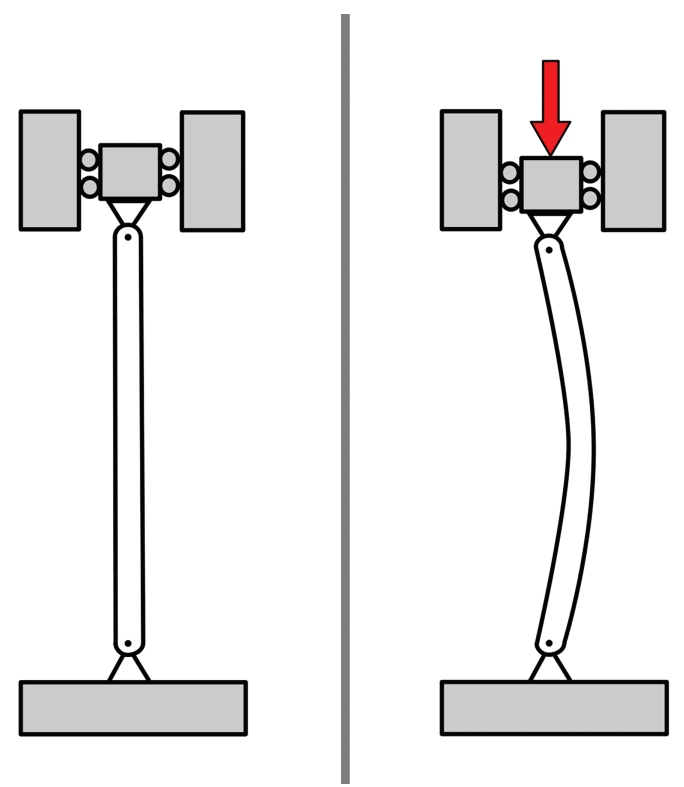

Figure 1. A rod in its original state (left), and buckled under compressive stress from a load (right).

This problem was studied in the 1750s by Euler, who established that the lateral deflection $w(x)$ at each point $x \in[0, L]$ in a rod with load $P$ solves the ODE

$$
E w^{\prime \prime}(x)+P w(x)=0,
$$

where $E>0$ is a physical constant determined by the rod's elasticity and the moments of inertia of its cross-sections. As one learns in an undergraduate course on ODEs, all solutions to (1) are of the form

$$
w(x)=A \sin (\lambda x)+B \cos (\lambda x), \quad \lambda=\sqrt{\frac{P}{E}},
$$

with $A, B \in \mathbb{R}$. In particular, if the rod is assumed to have pinned ends, that is, $w(0)=0=w(L)$, then it follows that $B=0$ and $\lambda=\lambda_{n}=\frac{n \pi}{L}$ for some nonnegative integer $n$. In other words, $w(x)$ is a boundary value problem eigenfunction (see Figure 2):

$$
w_{n}(x)=A \sin \left(\lambda_{n} x\right), \quad n=0,1,2, \ldots .
$$

Thus, for loads $P$ such that $\lambda<\lambda_{1}$, only the trivial solution $w_{0} \equiv 0$ exists. This means that the rod keeps its original shape if $P<E\left(\frac{\pi}{L}\right)^{2}$, and for this reason $P_{\max }=E\left(\frac{\pi}{L}\right)^{2}$ is called Euler's critical load. Meanwhile, for larger loads
$P>P_{\text {max }}$, such that $\lambda=\sqrt{\frac{P}{E}} \in\left[\lambda_{n}, \lambda_{n+1}\right)$, there are $n$ nontrivial solutions $w_{1}, \ldots, w_{n}$ to (1), which represent $n$ buckling modes that the rod may assume. These solutions can be seen as branches in the space of solutions to (1) that bifurcate from the trivial solution; see Figure 3.

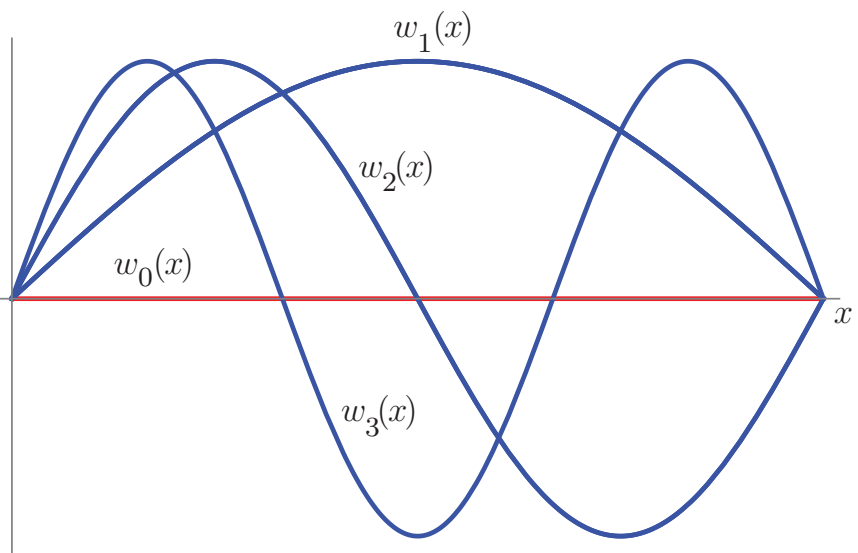

Figure 2. Graphs of $w_{n}(x)$ for $0 \leq n \leq 3$.

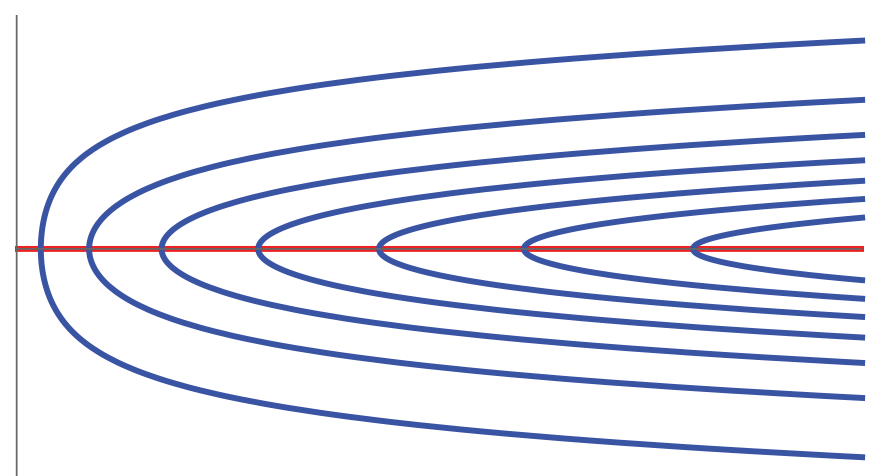

Figure 3. A bifurcation diagram with bifurcating branches (blue) issuing from the trivial branch (red). The horizontal axis represents the bifurcation parameter, which is the load $P$ in Euler's buckling problem. The trivial branch is $w_{0}(x) \equiv 0$, while the $n$th bifurcating branch, that exists for $P \geq E\left(\frac{n \pi}{L}\right)^{2}$, corresponds to $w_{n}(x), n \geq 1$. For each value of $P$, the number of different solutions to (1), up to rescaling by a positive factor, is the number of points where the vertical line through $P$ intersects the diagram. Note that this number grows to $+\infty$ as $P \nearrow+\infty$.

Deformations of structures due to buckling can be very serious and dangerous. For instance, train tracks may buckle and derail a train if they receive excessive heat from sun exposure or movement of trains; or external compression forces such as during an earthquake; see Figure 4. Although it would have been desirable from an engineer's perspective that no nontrivial solutions to (1) existed (so that no structures would buckle), from a mathematician's perspective, this bifurcation phenomenon can be used to 


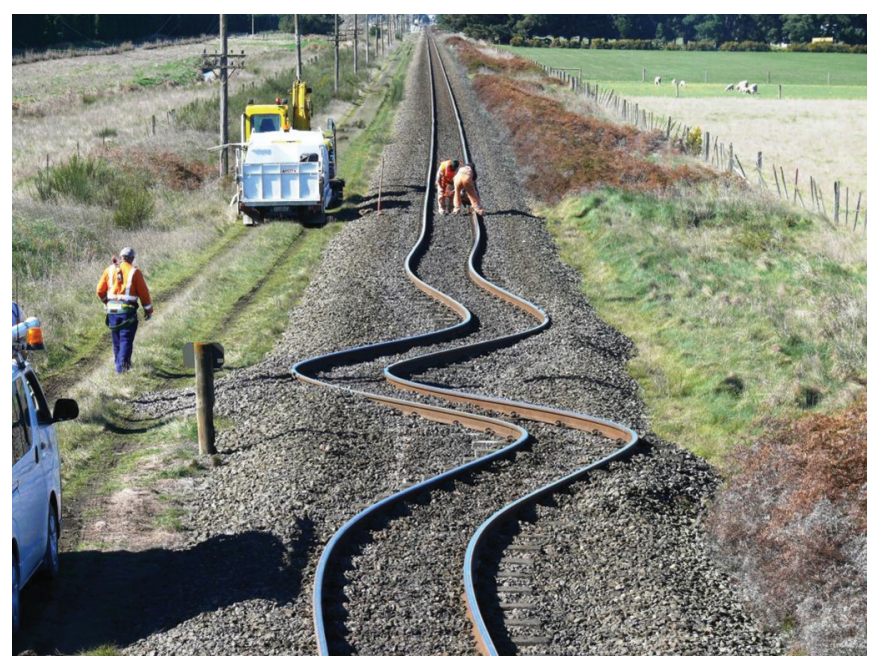

Figure 4. Train tracks in Canterbury, New Zealand, that buckled after an earthquake in 2010, in a shape that resembles the graph of $w_{2}(x)$.

produce new and otherwise hard-to-find solutions to certain problems. While the solutions (3) to the linear ODE (1) were easy to find and classify, solutions to nonlinear problems are generally much less accessible. It is in these situations that Bifurcation Theory is most useful, provided the equation at hand still has a readily available family of trivial solutions (for all values of the parameter), such as $w_{0} \equiv 0$ for (1). In this case, certain "tests" can be made on this family to detect the presence of bifurcations, and hence of previously unknown nearby solutions. We now describe some of these "tests" for variational equations.

\section{Bifurcation via Morse Index}

Let $X$ be the set of all states, or configurations, of a certain system, and assume $X$ is a (possibly infinite-dimensional) vector space or manifold. Suppose we are given a family $f_{t}: X \rightarrow \mathbb{R}$ of energy functionals on $X$, that we shall assume is sufficiently regular and parametrized by a real number $t \in[a, b]$. With it, the energy of each state $x \in X$ is measured to be $f_{t}(x)$, a quantity that depends on the parameter $t \in[a, b]$. Infinitesimal variations of $x \in X$ are described by tangent vectors to $X$ at $x$, which form a vector space denoted $T_{x} X$. This space typically comes equipped with a natural inner product $\langle\cdot, \cdot \cdot\rangle$. Stationary points, also called critical points, are solutions $x \in X$ to the EulerLagrange equation, or first variation equation:

$$
\mathrm{d} f_{t}(x)=0,
$$

where the derivative is in the variable $x$. We are interested in how solutions to (4) change with $t$. More precisely, say we are given a trivial branch of solutions, i.e., a curve $x_{t} \in X$, $t \in[a, b]$, satisfying $\mathrm{d} f_{t}\left(x_{t}\right)=0$ for all $t \in[a, b]$, and we would like to know whether (4) has any other solutions near $x_{t}$. So we are led to the following definition.
Definition 1. Bifurcation occurs at $t_{*}$ if there are converging sequences $x_{n} \rightarrow x_{t_{*}}$ in $X$ and $t_{n} \rightarrow t_{*}$, such that $\mathrm{d} f_{t_{n}}\left(x_{n}\right)=0$ and $x_{n} \neq x_{t_{n}}$; see Figure 5. In this situation, $t_{*}$ is called a bifurcation instant.

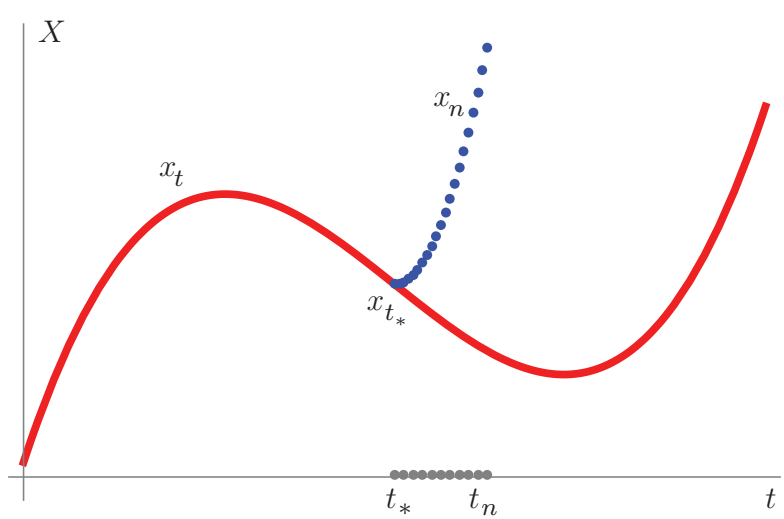

Figure 5. Graph of solutions to (4), i.e., a point $(t, x) \in[a, b] \times X$ is in the graph if and only if $\mathrm{d} f_{t}(x)=0$, with a bifurcation sequence (blue) $x_{n}$ issuing from the trivial branch (red) at $\left(t_{*}, x_{t_{*}}\right)$.

In other words, bifurcation occurs at $t_{*}$ if and only if the conclusion of the Implicit Function Theorem regarding equation (4) does not hold at $x_{t_{*}}$. Thus, its hypothesis must be violated, implying that a necessary condition for bifurcation to occur at $t_{*}$ is that $x_{t_{*}}$ be a degenerate critical point of $f_{t_{*}}$. Namely, the second derivative $\mathrm{d}^{2} f_{t_{*}}\left(x_{t_{*}}\right)$ cannot be invertible, i.e.,

$$
\operatorname{ker} J_{t_{*}}\left(x_{t_{*}}\right) \neq\{0\},
$$

where $J_{t}(x): T_{x} X \rightarrow T_{x} X$ is the self-adjoint linear operator that represents $\mathrm{d}^{2} f_{t}(x)$, in the sense that

$$
\mathrm{d}^{2} f_{t}(x)(\phi, \psi)=\left\langle J_{t}(x) \phi, \psi\right\rangle \quad \forall \phi, \psi \in T_{x} X .
$$

This operator $J_{t}(x)$ is called the Jacobi operator of this variational problem, which, if $X$ is finite dimensional, is just the Hessian matrix $\mathrm{d}^{2} f_{t}(x)$.

Nevertheless, (5) alone is not sufficient to guarantee that bifurcation occurs at $t_{*}$. A sufficient condition is given by a change in the degree of instability of $x_{t}$ as $t$ crosses $t_{*}$. This degree of instability is measured by an integer called the Morse index, defined for any solution $x$ to (4), as the number $i_{\text {Morse }}(x)$ of linearly independent directions at $x \in X$ that locally decrease the energy $f_{t}$. More precisely, denoting by $\operatorname{Spec}(\cdot)$ the set of eigenvalues of a linear operator,

$$
i_{\text {Morse }}(x)=\# \operatorname{Spec}\left(J_{t}(x)\right) \cap(-\infty, 0)
$$

is the number of negative eigenvalues of $J_{t}(x)$. (Note that $i_{\text {Morse }}(\cdot)$ depends on the parameter $t \in[a, b]$.)

Theorem 1 (Krasnosel'skii, 1964). If the trivial branch $x_{t} \in X$ of solutions to (4) is nondegenerate for all 
$t \in\left[t_{*}-\varepsilon, t_{*}+\varepsilon\right] \backslash\left\{t_{*}\right\}$ and $i_{\text {Morse }}\left(x_{t_{*}-\varepsilon}\right) \neq i_{\text {Morse }}\left(x_{t_{*}+\varepsilon}\right)$, then bifurcation occurs at $t_{*}$.

In the above statement, to simplify the exposition, we are omitting some technicalities (such as the requirement that $J_{t_{*}}\left(x_{t_{*}}\right)$ be Fredholm) that are important if $X$ is infinite dimensional; see [Kie12]. These conditions hold in the applications below.

Note that if $i_{\text {Morse }}\left(x_{t}\right)$ changes as $t$ crosses $t_{*}$, then $x_{t_{*}}$ must be degenerate; see (5). Indeed, a negative eigenvalue of $J_{t}\left(x_{t}\right)$ is gained or lost (so it must cross zero, by continuity) at $t=t_{*}$. In just a few words, the proof of Theorem 1 goes by contradiction: if no bifurcation occurred, then $\mathrm{d} f_{t}$ could be continuously deformed near $x_{t}$, between $t=t_{*}-\varepsilon$ and $t=t_{*}+\varepsilon$, keeping its (local) mapping degree constant; but this degree is precisely $i_{\text {Morse }}\left(x_{t}\right)$.

A toy problem from multivariable calculus. Let $X=\mathbb{R}^{2}$, and consider $f_{t}: X \rightarrow \mathbb{R}$,

$$
f_{t}(x, y)=\frac{1}{2}\left(x^{2}+y^{4}-t y^{2}\right) .
$$

Since $\mathrm{d} f_{t}(x, y)=\left(x, 2 y^{3}-t y\right)$, the solutions to (4) in this case are clearly $(x, y)=(0,0)$ for all $t \in \mathbb{R}$, and also $(x, y)=$ $(0, \pm \sqrt{t / 2})$ if $t \geq 0$.

Pretend, for a moment, we had not seen the latter solutions when $t \geq 0$, and only knew about the former (trivial) solutions $\left(x_{t}, y_{t}\right)=(0,0)$, which are easier to find, and exist for all $t \in \mathbb{R}$. From the Hessian matrix

$$
\mathrm{d}^{2} f_{t}(x, y)=\left(\begin{array}{cc}
1 & 0 \\
0 & 6 y^{2}-t
\end{array}\right)
$$

we compute the Morse index of $\left(x_{t}, y_{t}\right)=(0,0)$ to be:

$$
i_{\text {Morse }}(0,0)= \begin{cases}0, & \text { if } t \leq 0, \\ 1, & \text { if } t>0 .\end{cases}
$$

This jump of the Morse index at $t_{*}=0$ implies, by Theorem 1 , that $t_{*}=0$ is a bifurcation instant. Of course, the branch of solutions bifurcating from $(0,0)$ as $t$ crosses $t_{*}=0$ consists precisely of the solutions $(x, y)=(0, \pm \sqrt{t / 2})$ that we pretended not to see.

This can be illustrated with a bifurcation diagram (analogous to Figure 5), where the parameter $t$ is plotted along the horizontal axis, the vertical axis represents the space $X=\mathbb{R}^{2}$, and a point $(t, x, y)$ is in the graph if and only if it is a solution, i.e., $\mathrm{d} f_{t}(x, y)=0$; see Figure 6 . Note that for $t \leq 0$, the trivial solution $\left(x_{t}, y_{t}\right)=(0,0)$ is stable, since it is a local minimum of $f_{t}(x, y)$; actually its global minimum. However, for $t>0$, it is unstable, as it becomes a saddle point for $f_{t}(x, y), t>0$; see Figure 7 . This is essentially the same example Poincaré [Poi85] used as motivation.

Euler's buckling problem revisited. A quick look at Figures 3 and 6 suggests that the buckling phenomena could be interpreted as successive pitchfork bifurcations taking

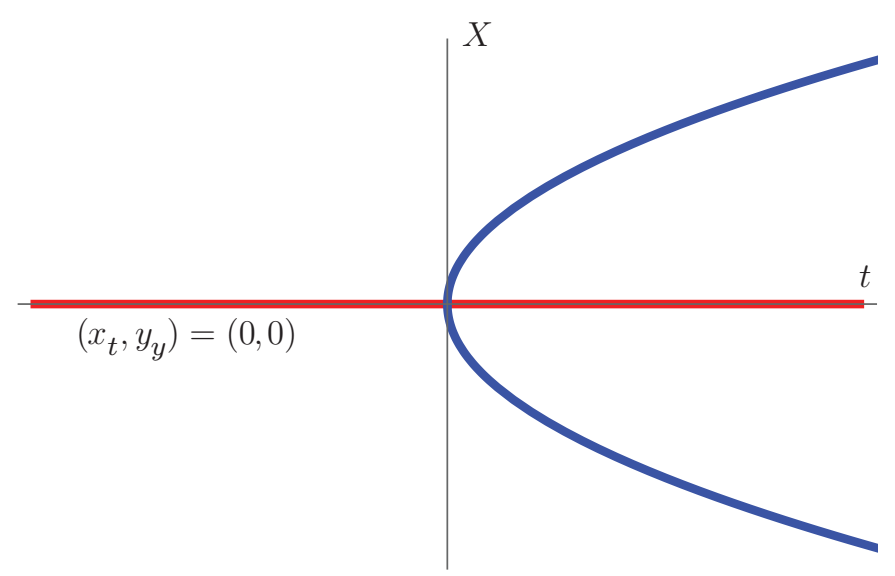

Figure 6. Bifurcation diagram for (6). Given the obvious resemblance, this is called pitchfork bifurcation.
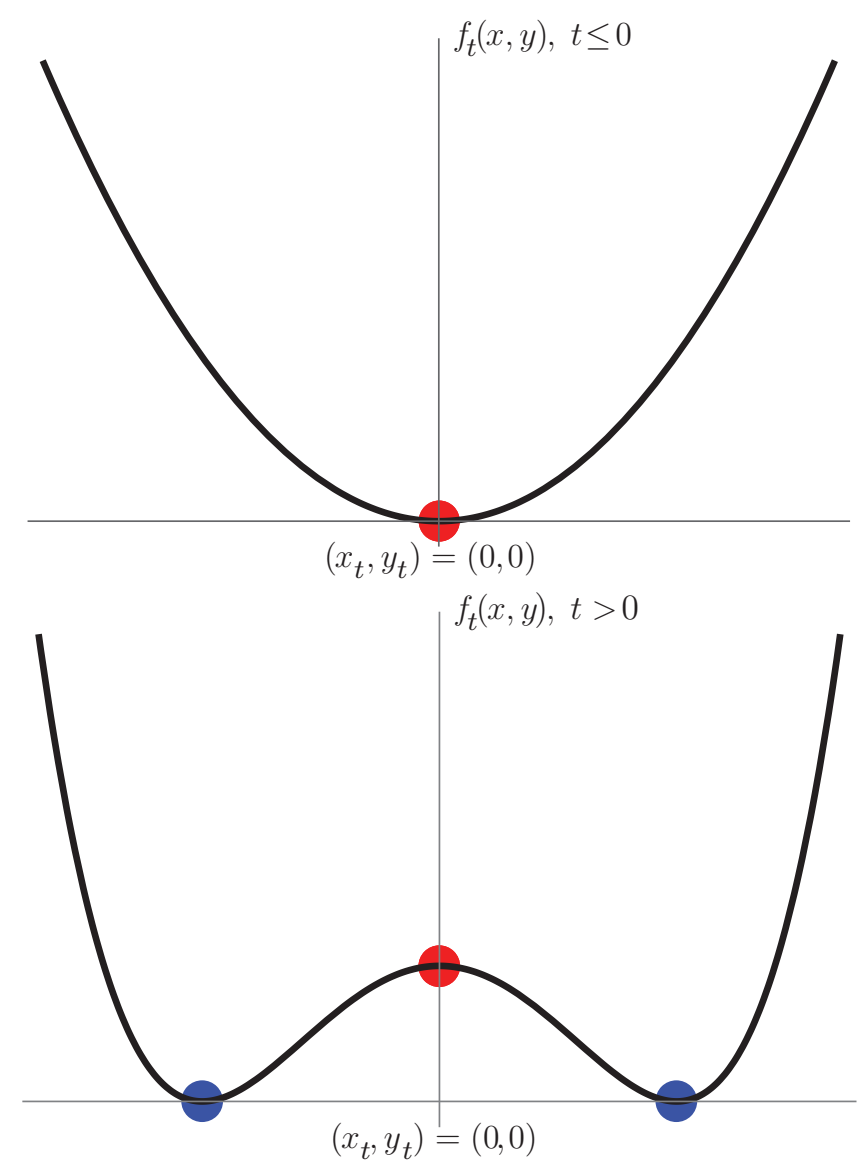

Figure 7. Schematic graphs of (6), where the horizontal axis represents $X=\mathbb{R}^{2}$. Note the change in stability of the critical point that belongs to the trivial branch (red) as $t$ crosses $t_{*}=0$. The critical points in the bifurcating branches (blue) are stable.

place as the load $P$ increases. We now show this is indeed the case.

Let $X$ be the space of functions $w:[0, L] \rightarrow \mathbb{R}$ that vanish at the endpoints, and consider the energy functional 
$f_{P}: X \rightarrow \mathbb{R}$ given by

$$
f_{P}(w)=\frac{1}{2} \int_{0}^{L} E\left(w^{\prime}\right)^{2}-P w^{2} \mathrm{~d} x,
$$

with parameter $P>0$. The first variation of $f_{P}$ is

$$
\begin{aligned}
\mathrm{d} f_{P}(w) u & =\int_{0}^{L} E w^{\prime} u^{\prime}-P w u \mathrm{~d} x \\
& =-\int_{0}^{L}\left(E w^{\prime \prime}+P w\right) u \mathrm{~d} x,
\end{aligned}
$$

from integration by parts and $w(0)=w(L)=0$. So, by the Fundamental Lemma of Calculus of Variations, $\mathrm{d} f_{P}(w)=$ 0 if and only if $w$ solves the ODE (1).

Observing that the constant $w_{0}(x) \equiv 0$ is a solution for all values of the parameter $P>0$, we are led to consider this as our trivial branch. Since the equation $\mathrm{d} f_{P}(w)=0$ is already linear in $w$ (cf. (8)), the Jacobi operator $J\left(w_{0}\right)$ of $w_{0}$ acts on $T_{w_{0}} X=X$ simply as

$$
J\left(w_{0}\right) \phi=-E \phi^{\prime \prime}-P \phi .
$$

Its eigenvalues are $E\left(\frac{n \pi}{L}\right)^{2}-P$ for all positive integers $n \in \mathbb{N}$. Thus,

$$
i_{\text {Morse }}\left(w_{0}\right)=\#\left\{n \in \mathbb{N}: E\left(\frac{n \pi}{L}\right)^{2}<P\right\}
$$

increases by 1 each time $P$ crosses a value of the form $P_{*}=E\left(\frac{n \pi}{L}\right)^{2}$. By Theorem 1, each of these is a bifurcation instant for the trivial branch $w_{0}$, matching the bifurcation diagram in Figure 3.

Due to its level of generality, this approach does not provide much more information about the bifurcating solutions apart from their existence. In particular, the explicit formula (3) is not recovered. However, it can be shown that the solutions bifurcating from $w_{0}$ form continuous (i.e., connected) branches.

Continuity of bifurcating branches. Recall that, from Definition 1, a bifurcating branch is just a sequence (and not necessarily a curve) of other solutions. But, strengthening the hypotheses of Theorem 1 to require that $i_{\text {Morse }}\left(x_{t}\right)$ changes exactly by 1 , it is possible to ensure the existence of an actual smooth curve of bifurcating solutions.

Theorem 2 (Crandall-Rabinowitz, 1971). If the trivial branch $x_{t} \in X$ of solutions to (4) is nondegenerate for all $t \in\left[t_{*}-\varepsilon, t_{*}+\varepsilon\right] \backslash\left\{t_{*}\right\}$, and only a simple eigenvalue $\lambda(t)$ of $J_{t}\left(x_{t}\right)$ crosses zero (transversely) at $t=t_{*}$, then solutions to (4) near $\left(t_{*}, x_{t_{*}}\right) \in \mathbb{R} \times X$ form two smooth curves that meet only at $\left(t_{*}, x_{t_{*}}\right)$.

Both the Calculus Toy Problem and Euler's Buckling Problem are situations where Theorem 2 applies (see (7) and (10)), yielding that bifurcating branches are continuous, as we had observed "by hand." Details on Theorem 2 and further refinements can be found in the textbook [Kie12].

\section{Geometric Variational Problems}

We now discuss some applications of Bifurcation Theory to geometric problems that are variational, i.e., take the form of the Euler-Lagrange equation (4) for some functional $f_{t}: X \rightarrow \mathbb{R}$, with parameter $t$.

These problems are naturally cast on Riemannian manifolds $M^{n}$, which are metric spaces made by (smoothly) patching together pieces of $\mathbb{R}^{n}$, where it is possible to measure lengths of vectors, compute angles, and, most importantly, use Differential and Integral Calculus. For instance, all smooth hypersurfaces $M^{n} \subset \mathbb{R}^{n+1}$ are automatically $n$ dimensional Riemannian manifolds, and, of course, so is $\mathbb{R}^{n}$ itself. Often, we omit the dimension $n$, writing $M$ for $M^{n}$.

Geodesics. One of the most elementary questions that can be posed on a Riemannian manifold $M$ is to find a curve joining two given points $p, q \in M$ with the least possible length. Such a curve always exists if $M$ is complete (e.g., if $M$ is compact), and is called a minimal geodesic. More generally, a geodesic is a curve that minimizes length between any two of its points, provided they are sufficiently close. Of course, geodesics in Euclidean space $\mathbb{R}^{n}$ are just straight lines. Similarly, geodesics on any Riemannian manifold $M$ are curves whose second derivative vanishes, or, in physical terms, trajectories traced by objects moving without acceleration. Thus, geodesics have constant speed. In Classical or Relativistic Optics, an optical medium is modeled by a Riemannian manifold, and trajectories of light rays are its geodesics.

Geodesics between $p, q \in M$ can be found using Calculus of Variations. Namely, they are stationary points of the energy functional $f: X \rightarrow \mathbb{R}$ given by

$$
f(\gamma)=\int_{0}^{1}\left|\gamma^{\prime}(s)\right|^{2} \mathrm{~d} s,
$$

where $X$ is the space of piecewise smooth curves $\gamma:[0,1] \rightarrow M$ with endpoints $\gamma(0)=p$ and $\gamma(1)=$ $q$. Moreover, $\gamma$ is a minimal geodesic if and only if it is a minimizer of $f$. As a technical aside, despite having (geometrically) the same curves as minimizers, (11) is more convenient to use than the actual length functional $\int_{0}^{1}\left|\gamma^{\prime}(s)\right| \mathrm{d} s$ because it is convex and its stationary points come parametrized with constant speed.

Infinitesimal variations of $\gamma$ are given by vector fields $V$ along $\gamma$, and represent the direction of the variation. In particular, variations of $\gamma$ within $X$, i.e., with fixed endpoints, are vector fields $V$ along $\gamma$ that vanish at the endpoints. The Jacobi operator $J(\gamma)$ of $\gamma$ acts on the space $T_{\gamma} X$ of such vector fields $V$ as

$$
J(\gamma) V=V^{\prime \prime}+R(V)
$$

where $R(V)$ is a curvature term, that is linear on $V$. 
Consequently, the Morse index of the geodesic $\gamma$ is

$$
i_{\text {Morse }}(\gamma)=\# \operatorname{Spec}(J(\gamma)) \cap(-\infty, 0) .
$$

It is very useful to also think of geodesics joining $p, q \in$ $M$ from a dynamic point of view. Namely, consider all curves with vanishing second derivative that depart from $p \in M$, and look for those that eventually pass through $q \in M$. With this approach, instead of minimizing (11) in the space $X$ of curves with endpoints $p, q \in M$, we analyze the solution $\gamma_{v}(t)$ to the geodesic ODE with initial position $\gamma_{v}(0)=p$ and initial velocity $\gamma_{v}^{\prime}(0)=v$, where $v \in T_{p} M$ is any tangent vector to $M$ at $p$. The collection of solutions to this geodesic initial value problem is conveniently encapsulated as the exponential map of $M$ at $p$,

$$
\exp _{p}: T_{p} M \rightarrow M, \quad \exp _{p}(t v)=\gamma_{v}(t) .
$$

Critical values of (14) are points in $M$ called conjugate to $p$, which are important for two main reasons.

First, dynamically, conjugate points to $p$ are points where a family of geodesics issuing from $p$ focalizes to first order. For instance, on the round sphere $S^{n} \subset \mathbb{R}^{n+1}$, geodesics are great circles. All the geodesics issuing from the North pole $p$ (called meridians, or longitude lines) focalize at the South pole $q$, which is hence conjugate to $p$. Moreover, this happens with multiplicity $n-1$, since the family of geodesics issuing from $p$ that focalizes at $q$ depends on $n-1$ independent parameters; for instance, in dimension $n=2$, meridians depend on only one parameter (the latitude). However, true focalization as in round spheres is very special; in general, focalization of geodesics at conjugate points occurs only to first order. Unfortunately, first-order phenomena are meaningless in very large scales, such as that of General Relativity models. In this context, more refined tools are needed to detect focalization, and we shall see below how Bifurcation Theory can provide them.

Second, conjugate points can be used to compute the Morse index $i_{\text {Morse }}(\gamma)$, defined in (13). Namely, if $\gamma$ is a geodesic starting at $p$, then $i_{\text {Morse }}(\gamma)$ is equal to the number of conjugate points to $p$ along $\gamma$, counted with multiplicity. In particular, if there are no conjugate points along $\gamma$, then $i_{\text {Morse }}(\gamma)=0$ and hence $\gamma$ minimizes length among nearby curves with the same endpoints. However, if $\gamma$ is sufficiently long, it might eventually develop conjugate points. This is triggered by curvature, manifested as $R(V)$ in the Jacobi operator (12), and related to the focalization phenomena described above. Nonpositive curvature keeps $i_{\text {Morse }}(\gamma)=0$ while traveling along $\gamma$, and it makes geodesics that started nearby diverge away, as straight lines in $\mathbb{R}^{n}$ or geodesics in the hyperbolic plane. On the other hand, positive curvature makes $i_{\text {Morse }}(\gamma)$ eventually increase, and geodesics focalize onto $\gamma$, as meridians on a round sphere. More precisely, by Theorem 1, bifurcation occurs when we cross the first conjugate point $q$ along $\gamma$, from which we deduce the existence of geodesics near $\gamma$ starting at $p$ and terminating shortly after $q$. Here, the bifurcation parameter $t$ is the distance travelled along $\gamma$, and the nondegeneracy hypothesis is satisfied because conjugate points on a geodesic are isolated.

Bifurcation of geodesics is well illustrated by the paraboloid $z=x^{2}+y^{2}$. Consider a geodesic $\gamma$ that starts at some point $p$ and passes through the origin; which is a "meridian" on this paraboloid. Due to positive curvature, some time after passing through the origin, $\gamma$ encounters a conjugate point $q$ to $p$. There, $i_{\text {Morse }}(\gamma)$ increases from 0 to 1 . This jump of the Morse index causes bifurcation, by Theorem 1 . In fact, the geodesics that constitute the bifurcating branch can be found explicitly: they also start at $p$ (but are not meridians) and meet $\gamma$ shortly after $q$, arbitrarily close to it; see Figure 8. Although these geodesics focalize at $q$ to first order, $\gamma$ is the unique geodesic joining $p$ to $q$. Since the Morse index jumps exactly by 1 , the assumptions of Theorem 2 are also satisfied here, so there is actually a continuum of bifurcating geodesics from $p$ focalizing at $q$. More generally, this happens whenever a simple (i.e., multiplicity 1) conjugate point is reached, which is always the case on surfaces due to dimensional reasons.
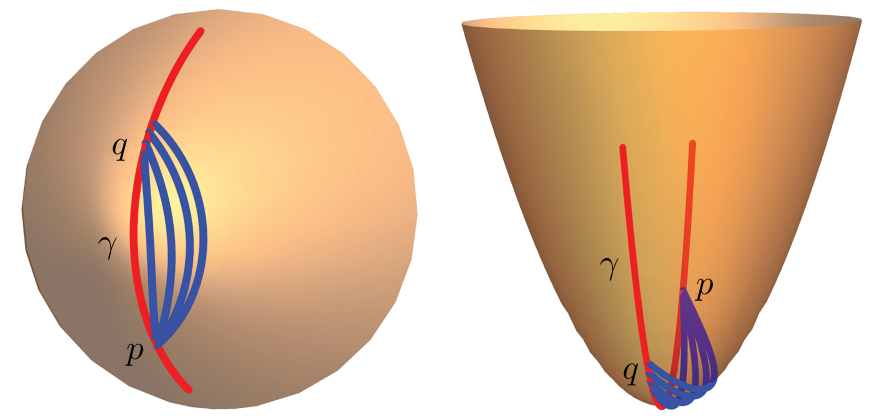

Figure 8. Geodesics on a paraboloid: a meridian $\gamma$ (red) and bifurcating geodesics (blue), issuing from $p$ and focalizing to first order at the conjugate point $q$.

Rephrasing the above discussion in terms of the exponential map (14) gives a bifurcation-theoretic proof of the following classical result.

Theorem 3 (Morse-Littauer, 1932). The exponential map is not locally injective near any critical point.

Indeed, from Definition 1 , if $v \in T_{p} M$ is a unit vector such that $t_{*} v$ is a critical point of $\exp _{p}$, then bifurcation gives a sequence $v_{n} \in T_{p} M$ of initial velocities converging to $t_{*} v$, with $v_{n} \neq t_{*} v$, such that $\exp _{p}\left(v_{n}\right)=\exp _{p}\left(t_{n} v\right)$ for some sequence $t_{n} \searrow t_{*}$. In other words, there exist pairs of distinct vectors, arbitrarily close to the critical point $t_{*} v$, which $\exp _{p}$ maps to the same point. Note this bifurcationtheoretic proof yields a slightly stronger conclusion: all these pairs can be chosen to include some multiple of $v$. 

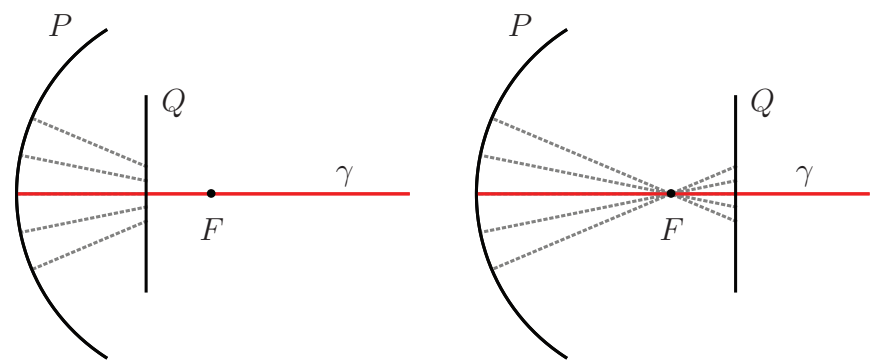

Figure 9. A focal point $F$ that does not produce bifurcation of orthogonal geodesics. The horizontal line segment $\gamma$ (red) is the unique geodesic in $\mathbb{R}^{2}$ departing orthogonally from the circular arc $P$ and arriving orthogonally at the vertical line segment $Q$. Crossing the focal point $F$ of $P$ at the center of the circle does not produce bifurcation, since the Morse index remains unchanged due to a cancellation phenomenon.

Geodesic bifurcation phenomena can also be studied with more general boundary conditions. One may replace the space $X$ of curves in $M$ from $p$ to $q$ with the space of curves that end at $q$, but whose initial endpoint is free to move along a given submanifold $P \subset M$. Stationary points for the energy (11) in this setup are geodesics that leave the initial submanifold $P$ orthogonally, and focalization of such geodesics occurs at so-called focal points of $P$, which are simply conjugate points if $P=\{p\}$. As in the fixed endpoint case, the occurrence of focal points produces bifurcation of geodesics. If the final endpoint is also left free to vary on a terminal submanifold $Q \subset M$, then an interesting cancellation phenomenon may happen, depending on the relative position of $P$ and $Q$. In this case, focal points do not correspond to jumps of the Morse index, and they do not necessarily produce bifurcation; see Figure 9 for an example from [GGP18].

Gravitational lensing. General Relativity uses fourdimensional spacetimes to model the universe, or parts of it. These are pseudo-Riemannian manifolds $\mathrm{M}^{4}$, where the length of nonzero vectors can be positive, negative, or null. Points in a spacetime are called events, since they not only represent the physical location of an "event," but also the time when it takes place. Vectors with negative length (called timelike vectors) represent velocities of objects with mass, and vectors with null length (called lightlike vectors) represent velocities of photons, which travel at the speed of light. Tangent lightlike vectors at an event form the socalled light cone of that event. Geodesics can also be defined here, although the notion of length extremality must be suitably reformulated. Consequently, the exponential map (14) carries over to the case in which $M$ is a general relativistic spacetime. Trajectories of light rays are geodesics in $M$ whose tangent vectors are everywhere lightlike, and the image of the light cone via the exponential map is the set of all light rays issuing from a light source located at the given event. The worldline of an observer is represented by a curve in $M$ whose tangent vector is everywhere timelike,

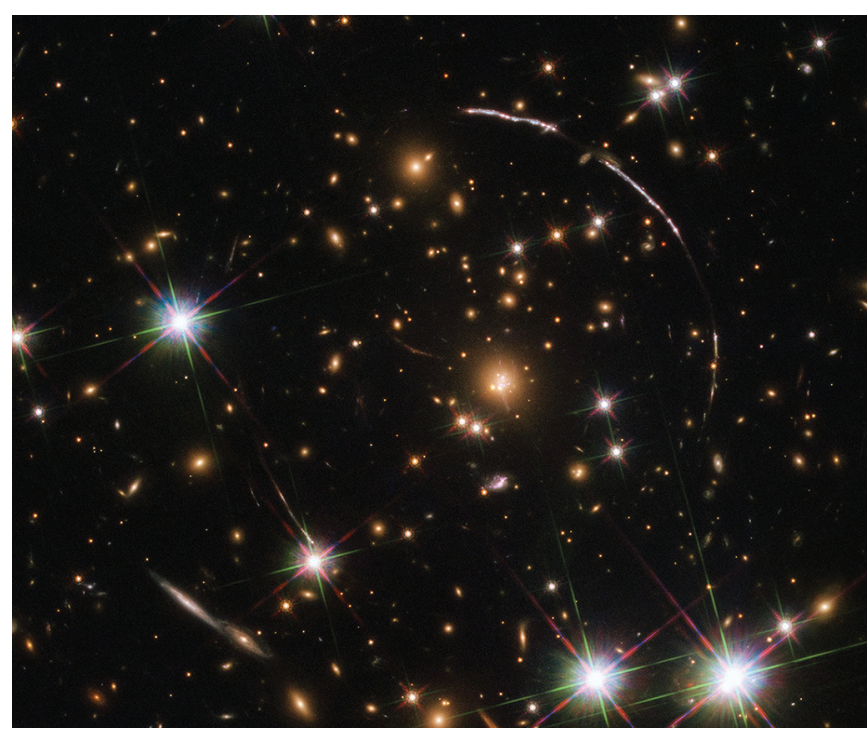

Figure 10. This picture from the NASA/ESA Hubble Space Telescope contains at least 12 simultaneous images (distributed over four arcs, three clearly visible on the top right corner) of the same light source, a galaxy (PSZ1 G311.65-18.48) almost 11 billion light-years from Earth. This lensing effect is due to a foreground cluster of galaxies 4.6 billion light-years away.

and the parameter of this curve is interpreted as the time measured by the observer.

To interpret bifurcation of geodesics representing light rays, consider all possible lightlike curves issuing from a given event $p \in M$, the light source; and a timelike curve $\gamma$, which is the worldline of an observer, say, a telescope in space. (The interpretation can also be reversed: the curve $\gamma$ may represent the worldline of a light source, and the event $p$ an instantaneous observer.) To each lightlike curve from $p$ to $\gamma$, i.e., each trajectory in spacetime with the speed of light from the light source to the observer one assigns its arrival time, as measured by the observer. The relativistic Fermat's Principle states that geodesics correspond exactly to minimizers, or more generally, stationary points for this arrival time function. Multiple lightlike geodesics from $p$ to $\gamma$ are interpreted as multiple images of the same instantaneous light source that the observer receives along its life (or, in the reverse interpretation, as images of the light source emitted at different stages of its life that are seen simultaneously by the observer). As in the case of Riemannian geodesics, this is an effect of curvature, which in a spacetime is caused by gravitational fields of large masses. Multiple images caused by such gravitational lensing were originally predicted by Einstein's theory of General Relativity in the 1930s, and first observed in the late 1970s; see Figure 10 for an example, and [Per00] and [GGP04] for details.

Constant mean curvature surfaces. One of the oldest problems in Mathematics is the isoperimetric problem of 


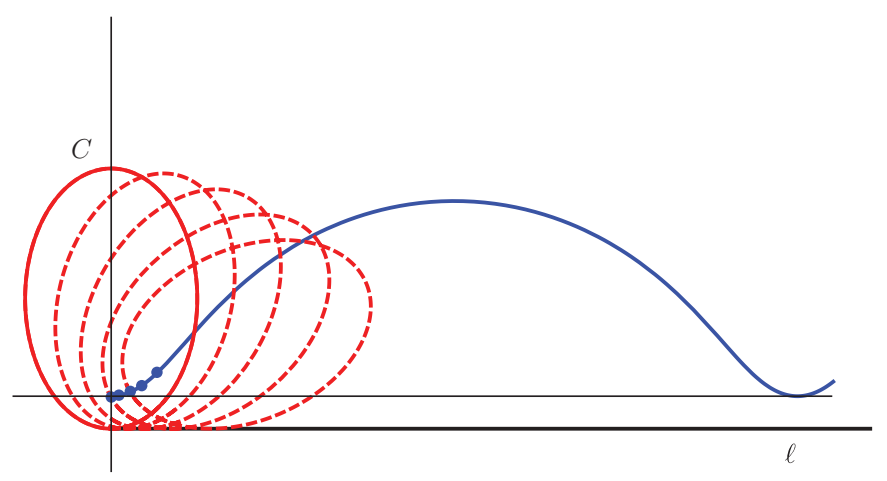

Figure 11. Roulette (blue) of a conic section (red).

determining the shape of a surface that has least area among surfaces bounding a given volume. These optimal contours, called isoperimetric surfaces, are the "preferred" configurations of many physical objects, such as soap bubbles. Solutions in Euclidean space are well known to be round spheres, but this problem becomes extremely challenging on more general ambient spaces. Although the existence and regularity of solutions on any $n$-dimensional Riemannian manifold $M$ has been established, there are very few ambients where all isoperimetric surfaces are explicitly known; see [Ros05] for a survey. For example, determining the isoperimetric surfaces in the complex projective plane $\mathbb{C} P^{2}$ remains an elusive open problem.

Using a Lagrange multiplier $H$ to deal with the volume constraint, we see that solutions are global minimizers of the functional $f_{H}: X \rightarrow \mathbb{R}$, given by

$$
f_{H}(x)=\operatorname{Area}(x)+H \operatorname{Vol}(x),
$$

where $x: \Sigma^{n-1} \hookrightarrow M^{n}$ is a hypersurface that encloses $n$ dimensional volume $\operatorname{Vol}(x)$, and has $(n-1)$-dimensional volume equal to $\operatorname{Area}(x)$. Of course, these quantities are named according to their more familiar meaning if the dimension of $M^{n}$ is $n=3$.

A possible strategy to solve the isoperimetric problem on $M^{n}$ is to first classify the hypersurfaces that are local minima of (15), i.e., solutions to $\mathrm{d} f_{H}(x)=0$ with $i_{\text {Morse }}(x)=0$, and then compare their volumes to search for the global minima. (For instance, this strategy was successfully used on sufficiently noncollapsed Berger spheres.) This also motivates studying all stationary points of (15), which are hypersurfaces that have constant mean curvature equal to $H$. Intuitively, these are shapes that, on average, bend the same amount $H$ in all directions, at all of its points. The special case in which $H=0$ is of great interest, since these are stationary points for the (unconstrained) area functional: they are called minimal hypersurfaces, and have been studied for centuries.

An infinitesimal perturbation of the hypersurface $x: \Sigma \hookrightarrow M$ is encoded as a function $\phi: \Sigma \rightarrow \mathbb{R}$, with $\phi(p)$ being the amount by which $x(p)$ is to be moved in the normal direction $\vec{n}_{x}(p)$. The Jacobi operator $J(x)$ of $x: \Sigma \hookrightarrow M$ acts on such a function $\phi$ as

$$
J(x) \phi=\Delta_{x} \phi-\left(\left\|A_{x}\right\|^{2}+\operatorname{Ric}\left(\vec{n}_{x}\right)\right) \phi,
$$

where $\Delta_{x}, A_{x}$, and $\operatorname{Ric}\left(\vec{n}_{x}\right)$ are respectively the Laplacian, the second fundamental form, and the Ricci curvature of $M$ in the direction normal to $x(\Sigma)$. In particular, $i_{\text {Morse }}(x)$ is the number of Laplace eigenvalues of the hypersurface $\Sigma$ that are smaller than $\left\|A_{x}\right\|^{2}+\operatorname{Ric}\left(\vec{n}_{x}\right)$. Both of these are curvature terms: $\left\|A_{x}\right\|^{2}$ encodes how $x(\Sigma)$ is curved inside $M$, while $\operatorname{Ric}\left(\vec{n}_{x}\right)$ measures the intrinsic curvature of $M$.

In 1841, Delaunay ingeniously classified surfaces of revolution in $\mathbb{R}^{3}$ that have constant mean curvature. Namely, he showed that their profile curve is the roulette of a conic section $C$, i.e., the curve traced by a focal point of $C$ as it rolls without slipping along a line $\ell$; see Figure 11. Revolving this roulette around $\ell$ produces a surface of revolution with constant mean curvature. These surfaces of revolution are called Delaunay surfaces and fall into three families according to which type of conic $C$ is used (see Figure 12):

- unduloids, if $C$ is an ellipse $(0<e<1)$;

- catenoids, if $C$ is a parabola $(e=1)$;

- nodoids, if $C$ is a hyperbola $(e>1)$;

aside from the limiting cases of spheres and cylinders.

Delaunay surfaces depend smoothly on the eccentricity $e>0$ and focal parameter $p>0$ of the conic $C$, and have constant mean curvature equal to

$$
H=\frac{\left|e^{2}-1\right|}{e p} .
$$

In particular, catenoids have $H=0$ and are the unique minimal surfaces of revolution in $\mathbb{R}^{3}$, apart from flat planes.

While all unduloids are stable, i.e., any other nearby constant mean curvature surface is again an unduloid, nodoids become increasingly unstable as $e \nearrow+\infty$, bifurcating infinitely many times. The resulting bifurcating branches consist of (topological) cylinders in $\mathbb{R}^{3}$ that have constant mean curvature, but are no longer surfaces of revolution. Instead, they are invariant under a discrete symmetry group; see Figure 13. This is an instance of symmetrybreaking bifurcation, where bifurcating solutions are less symmetric than those in the trivial branch.

As a side note, the careful reader might object that Delaunay surfaces in $\mathbb{R}^{3}$ (other than the sphere) have infinite area and enclose an infinite volume, hence (15) is not welldefined. Indeed, this variational characterization is only valid for hypersurfaces that bound a compact region. Nevertheless, the analysis can be reduced to a compact setting, adjusting $X$ accordingly, due to the periodic nature of Delaunay surfaces and of the bifurcating surfaces considered.

Bifurcation also provides a way to produce new Delaunay-type hypersurfaces in ambient spaces other than $\mathbb{R}^{3}$. For example, consider the 3-dimensional sphere 


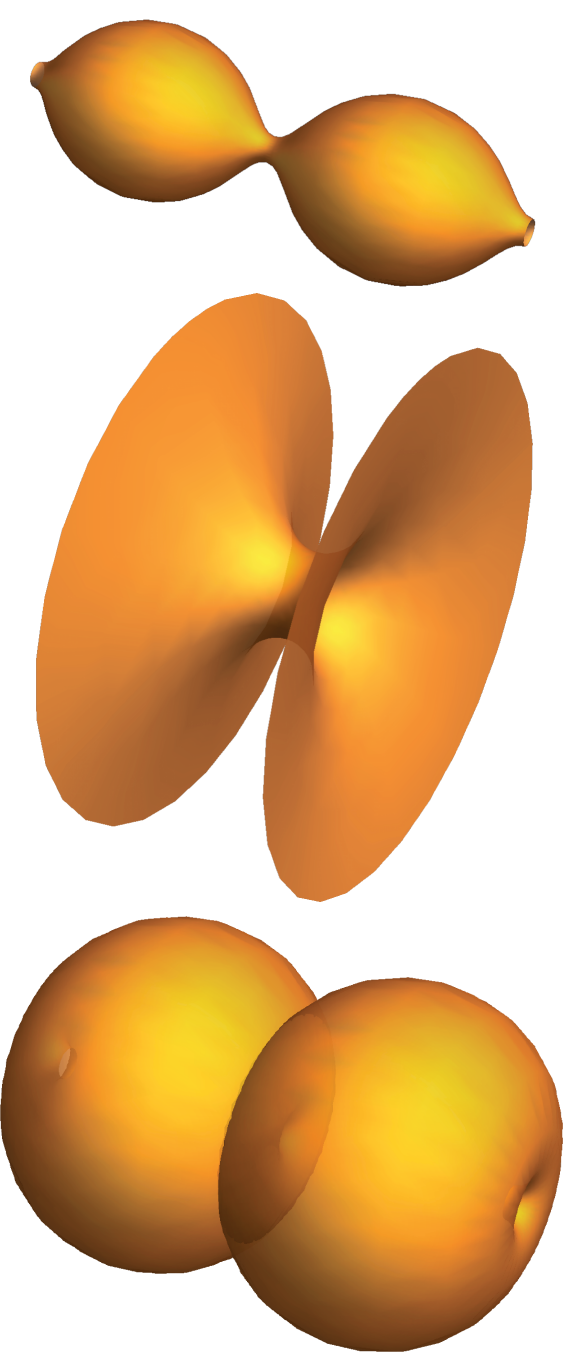

Figure 12. Compact portions of Delaunay surfaces (unduloid, catenoid, and nodoid, respectively) in $\mathbb{R}^{3}$.

$S^{3}=\left\{(z, w) \in \mathbb{C}^{2}:|z|^{2}+|w|^{2}=1\right\}$, and its partition into 2-tori $\Sigma_{t} \subset S^{3}$ for $t \in\left(0, \frac{\pi}{2}\right)$, given by

$$
\Sigma_{t}=\left\{(z, w) \in S^{3}:|z|=\cos t,|w|=\sin t\right\} .
$$

Note $\Sigma_{t}$ collapses to (Hopf-linked) great circles $\Sigma_{0}$ and $\Sigma_{\pi / 2}$ at the endpoints $t=0$ and $t=\frac{\pi}{2}$. The torus $\Sigma_{t}$ is the set of points at distance $t$ from $\Sigma_{0}$ in $S^{3}$, and has constant mean curvature equal to $H(t)=|\tan t-\cot t|$. In particular, $\Sigma_{\pi / 4}$ is the Clifford torus, which is notably the unique embedded minimal torus in $S^{3}$, up to rigid motions. Since the curvature term in (16) becomes unbounded as $t \searrow 0$ or $t \nearrow \frac{\pi}{2}$, while infinitely many eigenvalues of the Laplacian on $\Sigma_{t}$ remain bounded, we have that $i_{\text {Morse }}\left(\Sigma_{t}\right) \nearrow+\infty$, which, by Theorem 1 , yields the following.

Theorem 4. There are infinitely many branches of embedded constant mean curvature tori in $S^{3}$ that bifurcate from $\Sigma_{t}$ as $t \searrow 0$, and also as $t \nearrow \frac{\pi}{2}$.

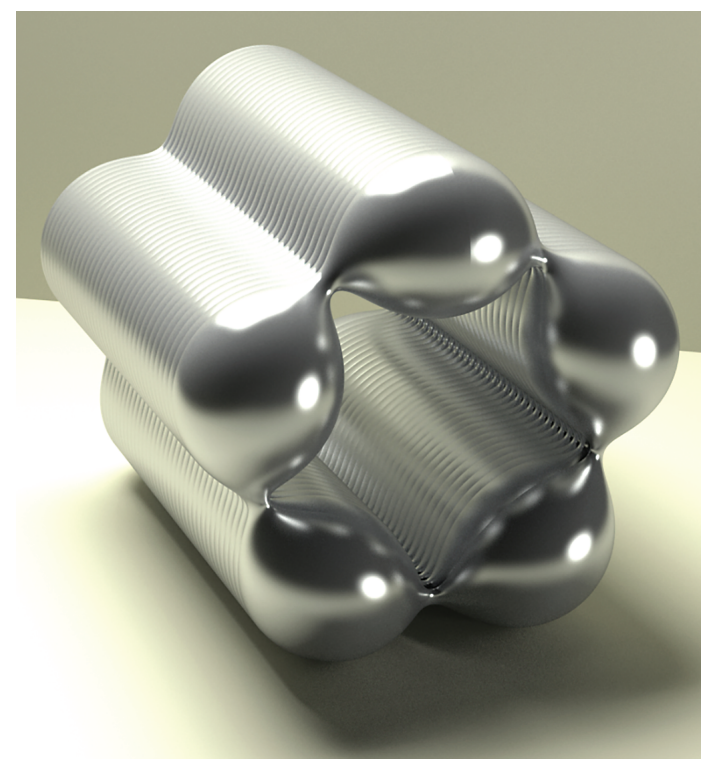

Figure 13. A surface with constant mean curvature in $\mathbb{R}^{3}$, found numerically, that bifurcates from a nodoid.

These bifurcating tori are Delaunay surfaces in $S^{3}$ (see Figure 14), and are actually its only embedded tori of constant mean curvature, up to rigid motions. This is another instance of symmetry-breaking, as the bifurcating tori have fewer symmetries than the trivial branch $\Sigma_{t}$. The same ideas can be used to construct Delaunay-type surfaces in much more general ambient spaces (cohomogeneity one manifolds), which include compact rank one symmetric spaces and even Kervaire exotic spheres [BP16].

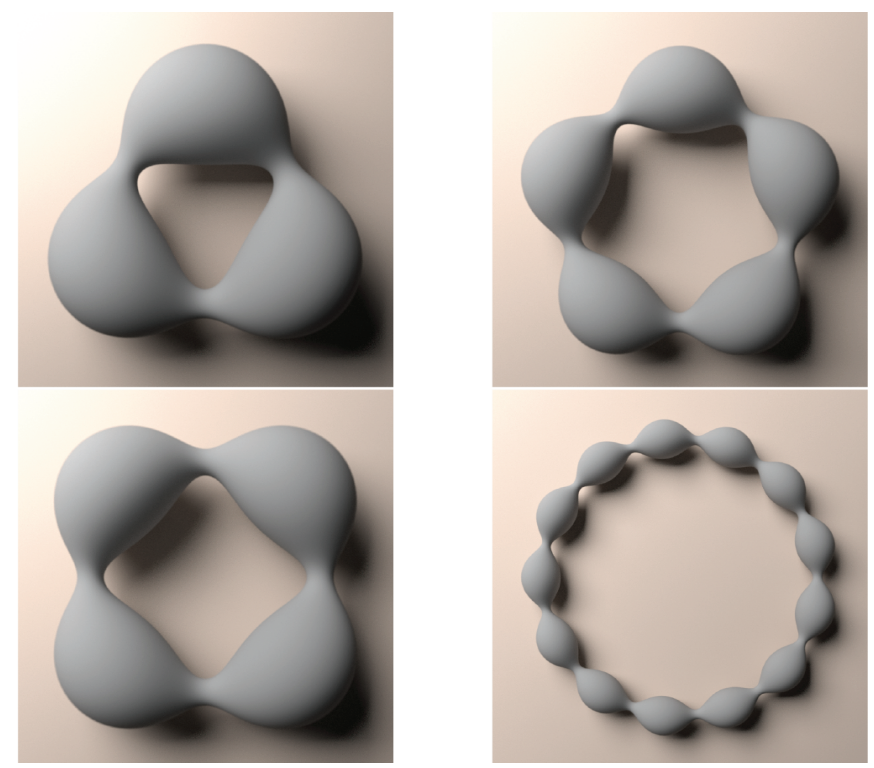

Figure 14. Delaunay tori in $S^{3}$ that bifurcate from $\Sigma_{t}$, viewed through stereographic projection to $\mathbb{R}^{3}$. 
Yamabe problem. A foundational result in Differential Geometry and Complex Analysis is the Uniformization Theorem, which originated with Klein and Poincare in the 1880s; see [dSG10] for a fascinating historic account. One of its many possible formulations states that every surface $M^{2}$ which is closed (i.e., compact and without boundary) can be deformed to have constant Gaussian curvature via a conformal transformation. This is a deformation in the way distances are measured that keeps all angles unchanged, but it may be difficult to visualize. For example, if $M^{2}$ is a surface in $\mathbb{R}^{3}$ which is not topologically a sphere, then the resulting conformally deformed surface of constant Gaussian curvature can no longer be embedded (isometrically) in $\mathbb{R}^{3}$. This deformation is also unique up to global rescalings, except again if $M^{2}$ is a sphere.

The Uniformization Theorem is inherently a 2-dimensional result, as it relies heavily on the intertwined nature of conformal deformations and complex structures in this dimension. In dimensions $n \geq 3$, one possible extension is to replace Gaussian curvature with scalar curvature, which at each $p \in M^{n}$ is an average of Gaussian curvatures of surfaces in $M^{n}$ through $p$. Using this notion, Yamabe proposed that every closed $n$-dimensional Riemannian manifold can be conformally deformed to have constant scalar curvature $c \in \mathbb{R}$. This corresponds to finding a positive solution $u: M^{n} \rightarrow \mathbb{R}$ to the PDE

$$
4 \frac{n-1}{n-2} \Delta_{g} u+\operatorname{scal}_{g} u=c u^{\frac{n+2}{n-2}}
$$

where $\operatorname{scal}_{g}: M^{n} \rightarrow \mathbb{R}$ is the original scalar curvature of $M^{n}$, and $\Delta_{g}$ its Laplacian (here, $g$ denotes the Riemannian metric of $M^{n}$ ). Given a solution, the desired conformal deformation is to multiply lengths of tangent vectors at each point $x \in M^{n}$ by $u(x)^{\frac{2}{n-2}}$. However, due to the value of the exponent causing the nonlinearity in the right-hand side of (17), standard existence results do not apply to such a PDE. Yamabe attempted to analyze it as a limit of PDEs with more favorable (subcritical) exponents in a paper published in 1960, but there were serious gaps in this approach. Tragically, he died of a stroke in that same year, at age 37. It took another 24 years for the result idealized by Yamabe to be eventually proven correct, as a combination of his work with that of Trudinger, Aubin, and Schoen; see [LP87] for a survey.

Although this completely solved the existence question, issues regarding uniqueness of solutions to the Yamabe problem, i.e., positive solutions to (17), remain an active area of research today. Using the maximum principle, it is easy to show that if (17) has a solution with $c \leq 0$, then it is the only solution. On the other hand, one typically expects nonuniqueness if $c>0$. A concrete instance of multiple solutions was described by Kobayashi and Schoen (independently), on the product $M_{t}^{n}$ of a sphere
$S^{n-1}$ of fixed radius and a circle $S^{1}(t)$ of varying radius $t$, as follows.

Theorem 5 (Kobayashi, Schoen). For all $n \geq 3$, the number of different solutions to the Yamabe problem on $M_{t}^{n}=S^{n-1} \times$ $S^{1}(t)$ tends to $+\infty$ as $t \nearrow+\infty$.

The Yamabe equation (17) is variational. Namely, its solutions are stationary points of the functional $f: X \rightarrow \mathbb{R}$ given by

$$
f(u)=\int_{M} 4 \frac{n-1}{n-2}\|\nabla u\|^{2}+\operatorname{scal}_{g} u^{2},
$$

where $X$ is the space of functions $u: M \rightarrow \mathbb{R}$ with

$$
\int_{M}|u|^{\frac{2 n}{n-2}}=1
$$

This constraint corresponds to a volume normalization of the conformal deformation. Aiming to prove nonuniqueness, let us assume that $M^{n}$ already has constant scalar curvature scal $g \equiv c$ and volume 1 , so that $u_{0} \equiv 1 \in X$ is a solution to (17), and seek other (nonconstant) solutions $u \in X$. Infinitesimal variations of $u_{0}$ in $X$ are identified with functions $v: M \rightarrow \mathbb{R}$ satisfying the linearized constraint $\int_{M} v=0$. Up to a dimensional constant, the Jacobi operator $J\left(u_{0}\right)$ of $u_{0} \equiv 1$ acts on such functions as

$$
J\left(u_{0}\right) v=\Delta_{g} v-\frac{\operatorname{scal}_{g}}{n-1} v .
$$

Consequently, its Morse index is

$$
i_{\text {Morse }}\left(u_{0}\right)=\# \operatorname{Spec}\left(\Delta_{g}\right) \cap\left(-\infty, \frac{\operatorname{scal}_{g}}{n-1}\right) .
$$

The general setup for bifurcation of solutions to the Yamabe problem is a 1-parameter family of Riemannian manifolds $M_{t}^{n}, t \in[a, b]$, with constant scalar curvature $\operatorname{scal}_{g(t)}>0$. If $\lambda(t)$ is a family of eigenvalues of the Laplacian $\Delta_{g(t)}$ of $M_{t}^{n}$ such that $\lambda(t)-\frac{\text { scal }_{g(t)}}{n-1}$ changes sign at $t=t_{*}$, then $i_{\text {Morse }}\left(u_{0}\right)$ jumps at $t=t_{*}$ and hence bifurcation occurs by Theorem 1 . If $\lambda(t)$ is a simple eigenvalue, then also Theorem 2 applies, implying continuity of the resulting bifurcating branch. Combined with other topological techniques, this can be used to show that the bifurcation diagram for $M_{t}^{n}=S^{n-1} \times S^{1}(t)$ is qualitatively the same as the one in Figure 3. This gives a bifurcationtheoretic proof of Theorem 5 .

Rescaling a factor in more general products also produces infinitely many bifurcations of solutions to the Yamabe problem. When closed manifolds $M$ and $N$ have constant scalar curvature, say $\operatorname{scal}_{M}>0$ and $\operatorname{scal}_{N}>0$, then so does $M \times N$, and $\operatorname{scal}_{M \times N}=\mathrm{scal}_{M}+\mathrm{scal}_{N}$. Furthermore, rescaling $N$ by $t>0$ produces a manifold $N_{t}$ with $\mathrm{scal}_{N_{t}}=\frac{1}{t} \mathrm{scal}_{N}$. Thus, $M \times N_{t}$ has constant scalar curvature $\operatorname{scal}_{M}+\frac{1}{t} \operatorname{scal}_{N}>0$. This quantity diverges to $+\infty$ as 
$t \searrow 0$, while infinitely many eigenvalues of the Laplacian remain bounded (those coming from $M$ ), so $i_{\text {Morse }}\left(u_{0}\right) \nearrow$ $+\infty$ by (20). Thus, from Theorem 1 , infinitely many bifurcations occur as $t \searrow 0$. Switching the roles of $M$ and $N$ corresponds to replacing $t$ with $1 / t$, and hence we arrive at the following.

Theorem 6. Infinitely many bifurcations occur for the Yamabe problem on $M \times N_{t}$ as $t \searrow 0$ or $t \nearrow+\infty$.

Generalizations of Theorem 6 from products to fiber bundles can be found in [BP13a, BP13b, OP].

The Yamabe problem can also be considered on noncompact manifolds $M^{n}$. In this situation, aside from being positive, a solution $u: M^{n} \rightarrow \mathbb{R}$ to (17) must diverge to $+\infty$ fast enough as one approaches infinity on $M^{n}$, so that the resulting conformal deformation is complete. Completeness can be thought of in the usual sense of metric spaces, i.e., Cauchy sequences must converge, and this plays the role of a boundary condition at infinity. Unlike the case of compact manifolds, the Yamabe problem on noncompact manifolds may have no solutions at all.

On the other hand, there are special cases where a solution can be built explicitly. For example, consider the complement $M^{n}=S^{n} \backslash S^{1}$ of a great circle in a round sphere $S^{n}, n \geq 3$. Since the round sphere has constant curvature scal $_{g}=n(n-1)$, so does the open subset $M^{n}$, but it is clearly not complete. However, rescaling $M^{n}$ by the appropriate (negative) power of the distance to its boundary, one obtains a complete Riemannian manifold which turns out to be conformally equivalent to the product $S^{n-2} \times H^{2}$, where $H^{2}$ is the hyperbolic plane. This product manifold has constant scalar curvature (because both of its factors do) equal to $(n-4)(n-1)$. Thus, we have found a solution to the Yamabe problem on $M^{n}$. Bifurcation Theory allows us to turn it into many more solutions.

Theorem 7. For all $n \geq 5$, there are uncountably many branches of (complete) solutions to the Yamabe problem on $S^{n} \backslash S^{1}$ conformal to the round metric.

We remark that the condition $n \geq 5$ is actually necessary for nonuniqueness of solutions, since it ensures that $c=(n-4)(n-1)>0$. In order to use Bifurcation Theory to prove Theorem 7 , we need a 1-parameter family of "trivial" solutions as the starting point, but so far we only have one solution, coming from the product $S^{n-2} \times H^{2}$. The trick to overcome this is to replace $H^{2}$ with compact quotients $H^{2} / \Gamma$, that is, hyperbolic surfaces. While there are uncountably many non-conformal hyperbolic surfaces, all of them are covered by the (same) hyperbolic plane $H^{2}$. Thus, new solutions to the Yamabe problem on $S^{n-2} \times H^{2} / \Gamma$ can be pulled back to $S^{n-2} \times H^{2}$, and then to $M^{n}=S^{n} \backslash S^{1}$. Since they come from a compact quotient, these solutions are automatically complete. Making a judicious choice of hyperbolic surfaces $H^{2} / \Gamma_{t}$ (see Figure 15), as $n \geq 5$, one can arrange for arbitrarily many eigenvalues of the Laplacian on $S^{n-2} \times H^{2} / \Gamma_{t}$ to become smaller than $\frac{\text { scal }_{g}}{n-1}=n-4$ as $t \searrow 0$. This forces $i_{\text {Morse }}\left(u_{0}\right) \nearrow+\infty$ by $(20)$, and hence infinitely many bifurcations by Theorem 1 . Pulling back the bifurcating solutions to $M^{n}$ as described above, one completes the proof of Theorem 7; see [BPS16] for details.
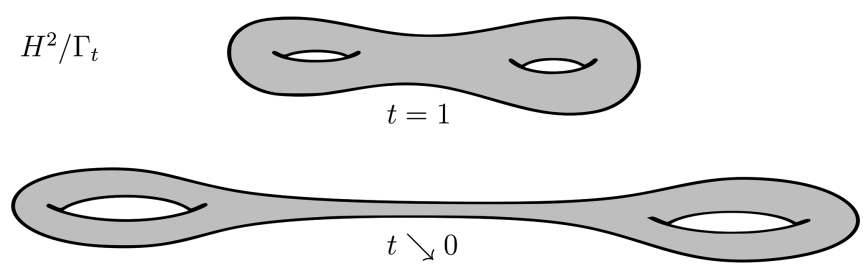

Figure 15. A curve $H^{2} / \Gamma_{t}$ of hyperbolic surfaces has the desired spectral behavior if its shortest nontrivial closed geodesic (systole) becomes arbitrarily small as $t \searrow 0$. Although the surfaces drawn above attempt to illustrate this, no hyperbolic surface can be actually seen (isometrically embedded) in $\mathbb{R}^{3}$.

\section{Generalizations}

Equivariant bifurcation. In many interesting situations, geometric variational problems have a nontrivial group of symmetries. More precisely, in terms of the abstract setup (4), suppose $f_{t}: X \rightarrow \mathbb{R}$ is invariant under the action of a group $\mathrm{G}$ of transformations of $X$; that is, $f_{t}$ is constant along G-orbits for all $t \in[a, b]$. In this situation, Theorem 1 cannot be applied if the stationary points $x_{t}$ along the trivial branch have nontrivial (i.e., nondiscrete) G-orbit, since tangent directions to them violate the nondegeneracy requirement.

However, Theorem 1 can be extended using a suitable equivariant nondegeneracy assumption, which requires the kernel of the Jacobi operator to be no larger than the tangent space to the G-orbit of the critical point. This is also the appropriate framework to study the symmetrybreaking phenomenon briefly mentioned above with Constant Mean Curvature hypersurfaces. This happens when the stabilizer subgroup of bifurcating solutions is smaller than that of solutions in the trivial branch. Symmetrybreaking also happens in the Yamabe problem; e.g., if the trivial branch consists of homogeneous manifolds, then every bifurcating solution is nonhomogeneous (since conformal homogeneous metrics are homothetic).

More sophisticated tools are available to detect bifurcation in equivariant problems. For instance, instead of only looking for jumps of the (equivariant) Morse index, replicating Theorems 1 and 2, finer representation-theoretic invariants of the negative eigenspaces of Jacobi operators can be used. For some details on these methods, see [SW90, BPS14].

Infinite Morse index. An essential assumption for the Bifurcation Theory discussed above is that the Morse index 
of all stationary points in the trivial branch is finite. This is always the case if the space of configurations $X$ is finite dimensional, but may fail in many interesting situations with infinite-dimensional $X$. Roughly speaking, finiteness of the Morse index is related to ellipticity of the Jacobi operator, such as in Euler's Buckling Problem (9), Riemannian geodesics (12), Constant Mean Curvature surfaces (16), and the Yamabe problem (19). Ellipticity implies that the negative part of the spectrum consists of finitely many eigenvalues, each with finite multiplicity, hence $i_{\text {Morse }}<$ $+\infty$.

Functional analytical techniques have been recently developed to study bifurcation in cases where the Morse index may be infinite. For instance, geodesics in manifolds endowed with a non-positive-definite (pseudoRiemannian) metric are stationary points with infinite Morse index; see for instance [AM05, AM08]. Although this is the case for general geodesics in spacetimes, we remark that the above discussion of gravitational lensing avoids this by using an alternative variational principle for light rays (with the arrival time functional) that yields stationary points of finite Morse index; see [GGP04]. More generally, infinite Morse index arises on stationary points of action functionals associated to nonconvex Lagrangians.

Despite remaining a largely unexplored area of Mathematics, important steps have been taken towards understanding a few special cases. A key notion that can replace the Morse index is the spectral flow, which is an integervalued invariant associated to paths of self-adjoint Fredholm operators on a Hilbert space. The spectral flow of a path gives a sort of algebraic count of how many eigenvalues cross 0 along the path, and can be defined in several different ways with tools from Functional Analysis. In some cases, bifurcation along a path of stationary points can be inferred from the corresponding path of Jacobi operators having nonzero spectral flow; see [FPR99].

To study bifurcation of pseudo-Riemannian geodesics, another key notion is the Maslov index of a geodesic $\gamma$, which is an integer that replaces the number of conjugate points (since they can accumulate along pseudoRiemannian geodesics). It is defined using symplectic techniques, passing to the Hamiltonian formulation, and linearizing the geodesic flow along $\gamma$. In this situation, jumps of the Maslov index detect bifurcation; see [PPT04].

ACKNOWLEDGMENTS. The first-named author is supported by the National Science Foundation (DMS1904342). The second-named author is supported by Fapesp (2016/23746-6 and 2019/09045-3).

\section{References}

[AM05] Alberto Abbondandolo and Pietro Majer, A Morse complex for infinite dimensional manifolds. I, Adv. Math. 197 (2005), no. 2, 321-410, DOI 10.1016/j.aim.2004.10.007 MR2173848

[AM08] Alberto Abbondandolo and Pietro Majer, A Morse complex for Lorentzian geodesics, Asian J. Math. 12 (2008), no. 3, 299-319, DOI 10.4310/AJM.2008.v12.n3.a3 MR2453558

[BP13a] Renato G. Bettiol and Paolo Piccione, Bifurcation and local rigidity of homogeneous solutions to the Yamabe problem on spheres, Calc. Var. Partial Differential Equations 47 (2013), no. 3-4, 789-807, DOI 10.1007/s00526-012-0535y MR3070564

[BP13b] Renato G. Bettiol and Paolo Piccione, Multiplicity of solutions to the Yamabe problem on collapsing Riemannian submersions, Pacific J. Math. 266 (2013), no. 1, 1-21, DOI 10.2140/pjm.2013.266.1. MR3105774

[BP16] Renato G. Bettiol and Paolo Piccione, Delaunaytype hypersurfaces in cohomogeneity one manifolds, Int. Math. Res. Not. IMRN 10 (2016), 3124-3162, DOI 10.1093/imrn/rnv231. MR3551832

[BPS16] Renato G. Bettiol, Paolo Piccione, and Bianca Santoro, Bifurcation of periodic solutions to the singular Yamabe problem on spheres, J. Differential Geom. 103 (2016), no. 2, 191-205. MR3504948

[BPS14] Renato G. Bettiol, Paolo Piccione, and Gaetano Siciliano, Equivariant bifurcation in geometric variational problems, Analysis and topology in nonlinear differential equations, Progr. Nonlinear Differential Equations Appl., vol. 85, Birkhäuser/Springer, Cham, 2014, pp. 103-133. MR3330725

[dSG10] Henri Paul de Saint-Gervais, Uniformisation des surfaces de Riemann (French), ENS Éditions, Lyon, 2010. Retour sur un théorème centenaire. [A look back at a 100year-old theorem]. MR2768303

[FPR99] Patrick M. Fitzpatrick, Jacobo Pejsachowicz, and Lazaro Recht, Spectral flow and bifurcation of critical points of strongly-indefinite functionals. I. General theory, J. Funct. Anal. 162 (1999), no. 1, 52-95, DOI 10.1006/jfan.1998.3366 MR1674534

[GGP04] Roberto Giambò, Fabio Giannoni, and Paolo Piccione, Gravitational lensing in general relativity via bifurcation theory, Nonlinearity 17 (2004), no. 1, 117-132, DOI 10.1088/0951-7715/17/1/008, MR2023435

[GGP18] Roberto Giambò, Fabio Giannoni, and Paolo Piccione, A finite dimensional approach to light rays in general relativity, Nonlinear Anal. 168 (2018), 198-221, DOI 10.1016/j.na.2017.11.014. MR3759476

[Kie12] Hansjörg Kielhöfer, Bifurcation theory: An introduction with applications to partial differential equations, 2nd ed., Applied Mathematical Sciences, vol. 156, Springer, New York, 2012. MR2859263

[LP87] John M. Lee and Thomas H. Parker, The Yamabe problem, Bull. Amer. Math. Soc. (N.S.) 17 (1987), no. 1, 37-91, DOI 10.1090/S0273-0979-1987-15514-5. MR888880

[OP] Nobuhiko Otoba and Jimmy Petean, Bifurcation for the constant scalar curvature equation and harmonic Riemannian submersions, J. Geom. Anal. to appear. 
[Per00] Volker Perlick, Gravitational lensing from a geometric viewpoint, Einstein's field equations and their physical implications, Lecture Notes in Phys., vol. 540, Springer, Berlin, 2000, pp. 373-425, DOI 10.1007/3-540-465804_6. MR1765134

[PPT04] Paolo Piccione, Alessandro Portaluri, and Daniel V. Tausk, Spectral flow, Maslov index and bifurcation of semi-Riemannian geodesics, Ann. Global Anal. Geom. 25 (2004), no. 2, 121-149, DOI 10.1023/B:AGAG.0000018558.65790.db MR2046769

[Poi85] H. Poincaré, Sur l'équilibre d'une masse fluide animée d'un mouvement de rotation (French), Acta Math. 7 (1885), no. 1, 259-380, DOI 10.1007/BF02402204 MR1554685

[Ros05] Antonio Ros, The isoperimetric problem, Global theory of minimal surfaces, Clay Math. Proc., vol. 2, Amer. Math. Soc., Providence, RI, 2005, pp. 175-209. MR2167260

[SW90] J. Smoller and Arthur G. Wasserman, Bifurcation and symmetry-breaking, Invent. Math. 100 (1990), no. 1, 63-95, DOI 10.1007/BF01231181. MR1037143

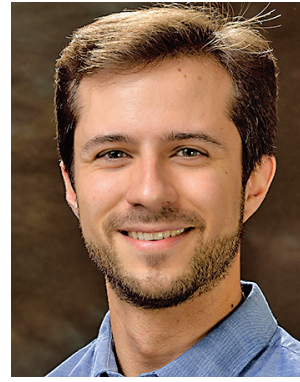

Renato G. Bettiol

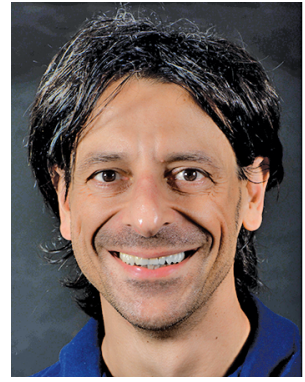

Paolo Piccione

\section{Credits}

The opening image is courtesy of Jukrachai Inthidech via Getty.

Figure 1 is from Wikimedia (public domain).

Figure 4 is courtesy of Malcolm Teasdale (Kiwi Rail) from 2010.

Figure 10 is courtesy of NASA, ESA, and E. Rivera-Thorsen (Institute of Theoretical Astrophysics, Oslo, Norway).

Figures 13 and 14 are courtesy of Nicholas Schmidt with xlab@GeometrieWerkstatt.

All other figures are courtesy of the authors.

Photo of Renato G. Bettiol is courtesy of Felice Macera.

Photo of Paolo Piccione is courtesy of Baú da Lola.

\section{FEATURED TITLES FROM THE}

EUROPEAN MATHEMATICAL SOCIETY

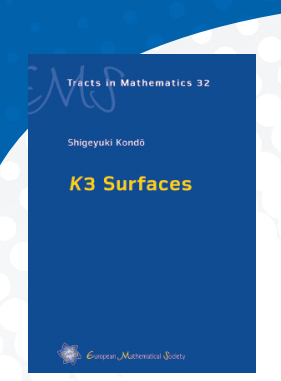

K3 Surfaces

Shigeyuki Kondō, Nagoya University, Japan

$K 3$ surfaces are a key piece in the classification of complex analytic or algebraic surfaces. The term was coined by A. Weil in 1958-a result of the initials Kummer, Kähler, Kodaira, and the mountain $K 2$ found in Karakoram. The most famous example is the Kummer surface discovered in the 19th century. K3 surfaces can be considered as a 2-dimensional analogue of an elliptic curve, and the theory of periods-called the Torelli-type theorem for $K 3$ surfaces-was established around 1970

EMS Tracts in Mathematics, Volume 32; 2020; 250 pages; Hardcover; ISBN: 978-3-03719-208-5; List US $\$ 88$; AMS members US $\$ 70.40$; Order code EMSTM/32

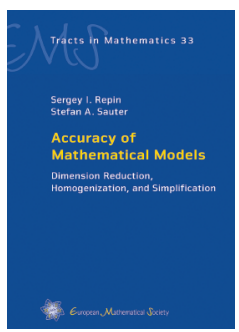

\section{Accuracy of} Mathematical Models

Dimension Reduction, Homogenization, and Simplification

Sergey Repin, Russian Academy of Sciences, St. Petersburg and Stefan Sauter, University of Zürich, Switzerland

The expansion of scientific knowledge and the development of technology are strongly connected with quantitative analysis of mathematical models. Accuracy and reliability are the key properties we wish to understand and control

This book presents a unified approach to the analysis of accuracy of deterministic mathematical models described by variational problems and partial differential equations of elliptic type. It is based on new mathematical methods developed to estimate the distance between a solution of a boundary value problem and any function in the admissible functional class associated with the problem in question. The theory is presented for a wide class of elliptic variational problems. It is applied to the investigation of modelling errors arising in dimension reduction, homogenization, simplification, and various conversion methods (penalization, linearization, regularization, etc.). A collection of examples illustrates the performance of error estimates.

EMS Tracts in Mathematics, Volume 33; 2020; 333 pages; Hardcover; ISBN: 978-3-03719-206-1; List US\$65; AMS members US\$52; Order code EMSTM/33 